\title{
Combination of Osimertinib and Anlotinib May Overcome the Resistance Mediated by in cis EGFR T790M-C797S in NSCLC: A Case Report
}

\author{
Rengui Zhou' \\ Lei Song' \\ Wenwen Zhang' \\ Lin Shao ${ }^{2}$ \\ $\mathrm{Xi} \mathrm{Li}{ }^{2}$ \\ Xiangyong $\mathrm{Li}^{\prime}$ \\ 'Department of Hematology and \\ Oncology, The 904 Hospital of the PLA \\ (Chinese People's Liberation Army), \\ Wuxi, People's Republic of China; \\ ${ }^{2}$ Burning Rock Biotech, Guangzhou, \\ Guangdong, People's Republic of China
}

Correspondence: Xiangyong Li Department of Hematology and Oncology, The 904 Hospital of The PLA (Chinese People's Liberation Army), North XingYuan Road NO.I0I, Wuxi, Jiangsu, 214044, People's Republic of China

Email xiangyongl@।26.com

\begin{abstract}
The emergence of epidermal growth factor receptor (EGFR) exon 20 p.C797S is one of the major resistance mechanisms for osimertinib. However, there are no standard of care for non-small cell lung cancer (NSCLC) patients after acquiring EGFR C797S currently, which brings significant challenges to post-osimertinib clinical management. In the present study, we described a 52-year-old female patient with EGFR-mutated stage IV lung adenocarcinoma, who achieved a partial response (PR) to the treatment of gefitinib and osimertinib after acquiring EGFR exon 20 p.T790M-trans-C797S at osimertinib failure. After progression on the combinatorial treatment, allelic configuration shifted to T790M-cis-C797S. The patient subsequently received a regimen of osimertinib and anlotinib combined with chemotherapy, followed by osimertinib and anlotinib maintenance treatment, and achieved a PR lasting for 9 months. At disease progression, concomitant T790M-C797S mutations both in trans and cis were identified. A combination of chemo- and anti-angiogenic therapies was administrated for two cycles and then discontinued because of the poor physical condition of the patient. She passed away soon with an overall survival of 39 months and a postosimertinib progression survival of 20 months. Our study provides the first clinical evidence that the osimertinib and anlotinib-based regimen may be an effective therapy in overcoming resistance mediated by T790M-cis-C797S. Our case also highlights the importance of dynamically monitoring the mutation status after osimertinib failure, which may provide patients with increased opportunities for targeted therapy and improve post-osimertinib progression survivals.
\end{abstract}

Keywords: EGFR TKI, EGFR T790M, EGFR C797S, NSCLC, osimertinib, anlotinib

\section{Introduction}

Most epidermal growth factor receptor (EGFR)-mutant non-small cell lung cancer (NSCLC) patients with initial responsiveness to EGFR tyrosine kinase inhibitors (TKIs) will inevitably acquire drug resistance. Secondary EGFR exon20 p.T790M (T790M) is the most common mechanism mediating the resistance to firstand second-generation TKIs. ${ }^{1}$ The third-generation EGFR TKI osimertinib selectively targets both activating EGFR mutations and T790M. Despite its robust clinical efficacy and promising survival advantages, osimertinib-treated patients ultimately develop secondary resistance with the emergence of EGFR exon20 p.C797S (C797S) in cis or trans with T790M as the major mechanism. ${ }^{2}$ In cis and in trans configuration refers to that $\mathrm{C} 797 \mathrm{~S}$ is present on the same and different EGFR allele as T790M, respectively. ${ }^{3}$ Unfortunately, there is no standard of care for EGFR-mutant NSCLC 
patients after acquiring C797S, which brings significant challenges to post-osimertinib clinical management. T790Mtrans-C797S, which occurs in $\sim 10 \%$ of C797S cases, has been reported to respond to the combination of first and third-generation EGFR-TKIs. ${ }^{4}$ Whereas, T790M-cis$\mathrm{C} 797 \mathrm{~S}$, the more frequent allelic configuration accounting for $\sim 80 \%$ of $\mathrm{C} 797 \mathrm{~S}$ cases, ${ }^{5}$ lacks effective targeted therapy.

Here, we reported a patient with stage IV lung adenocarcinoma, who achieved a partial response (PR) to the treatment of gefitinib combined with osimertinib after acquiring T790M-trans-C797S at osimertinib failure. After progression on combinatorial treatment, allelic configuration shifted to T790M-cis-C797S. The patient subsequently received a regimen of osimertinib and anlotinib combined with chemotherapy and achieved a sustained response of 9 months.

\section{Case Presentation}

The patient's diagnostic and treatment history was summarized in Figure 1A. The 52-year-old female presented to our hospital in June 2017, with coughing of phlegm for 1 month and a PS score of 1. Chest computed tomography (CT) scan revealed consolidation in right lower lung accompanied with right pleural effusion and scattered miliary opacities on both lungs (Figure 1B). The patient subsequently underwent a bronchofibroscopy and was diagnosed with stage IV (cTxNxM1) adenocarcinoma histopathologically. An amplification refractory mutation system polymerase chain reaction (ARMS-PCR) test identified an EGFR exon 19 deletion (19 del) in tissue. Gefitinib was administrated as first-line treatment and the patient achieved a partial response (PR) with a progression-free survival (PFS) of 7 months. At gefitinib progression, next-generation sequencing (NGS) using a 520-gene panel (Burning Rock Biotech, China) was performed with the patient's plasma sample and revealed the retention of EGFR 19 del (p. E746_A750del, allele frequency (AF):0.07\%) and the emergence of T790M (AF:0.16\%). The patient was subsequently switched to osimertinib in Feb 2018 and achieved a PR with a PFS of 10 months.

In Dec 2018, the patient developed disease progression (PD) with an enlarged lesion on the right lung, increased volume of pleural effusion, and atelectasis of the right lobe, revealed by chest CT scan (Figure 1C). NGS using the same panel was performed with plasma and pleural effusion samples and revealed EGFR 19del and T790Mtrans-C797S in both samples (Figure 2A). The AF of plasma $19 \mathrm{del}$ and $\mathrm{T} 790 \mathrm{M}$ increased to $0.24 \%$ and $0.20 \%$, respectively. $\mathrm{C} 797 \mathrm{~S}$ had an $\mathrm{AF}$ of $0.38 \%$. In Jan 2019, the patients started third-line treatment of osimertinib (80mg/QD) combined with gefitinib (250 mg/ QD) and achieved a PR one month later with shrinkage of right lung lesion and reduction of pleural effusion

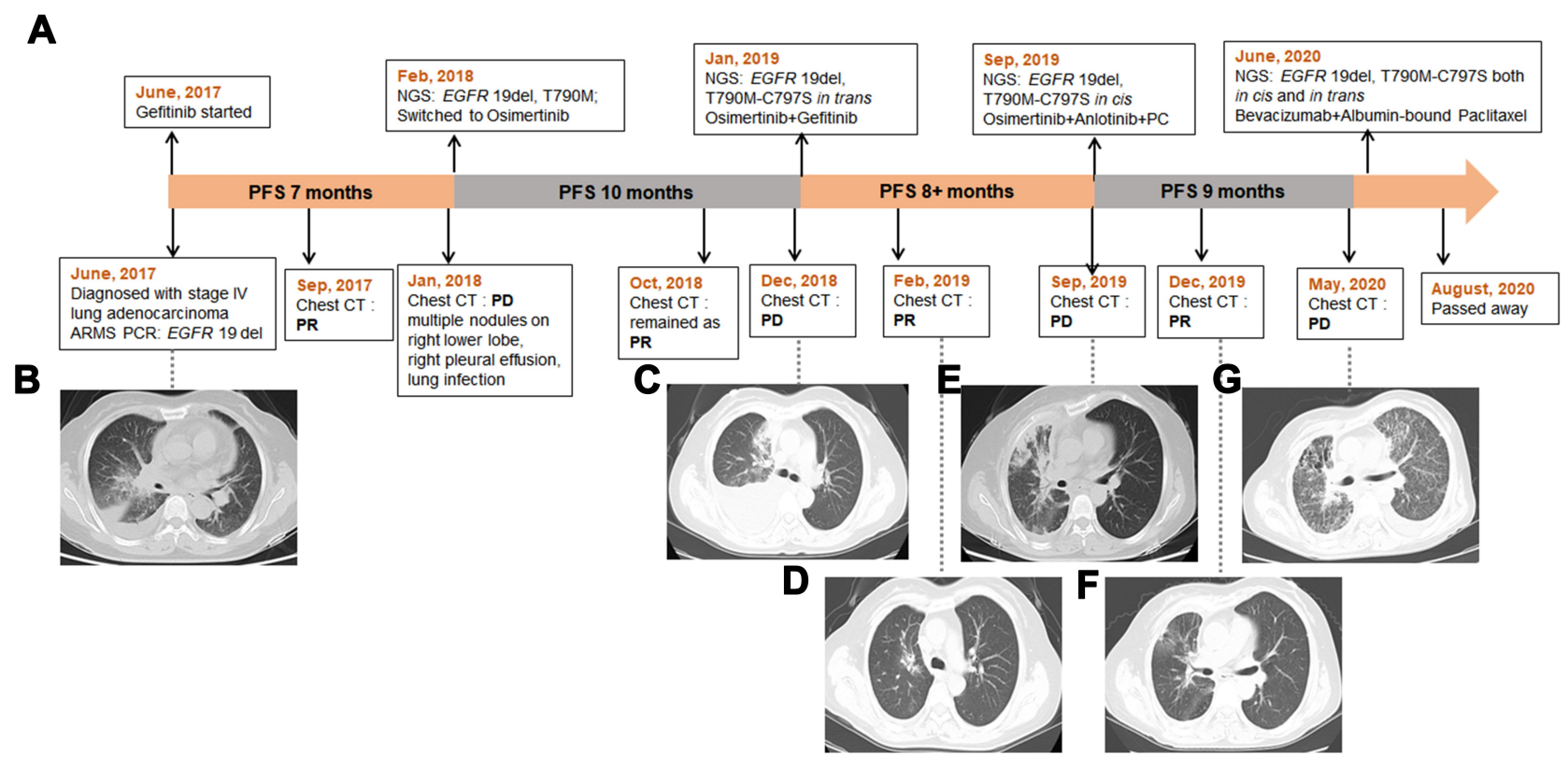

Figure I The timeline of the patient's treatment history and the response of the tumor lesions. (A) the timeline of treatments; (B) the chest CT image at initial diagnosis; (C) disease progression (PD) on osimertinib; (D) partial response (PR) to osimertinib+ gefitinib; (E) PD on osimertinib+ gefitinib; (F) PR to the treatment of osimertinib+ anlotinib+ chemotherapy; (G) PD on osimertinib+ anlotinib+ chemotherapy.

Abbreviations: ARMS-PCR, amplification refractory mutation system polymerase chain reaction; NGS, next-generation sequencing; PC, pemetrexed+ cisplatin. 

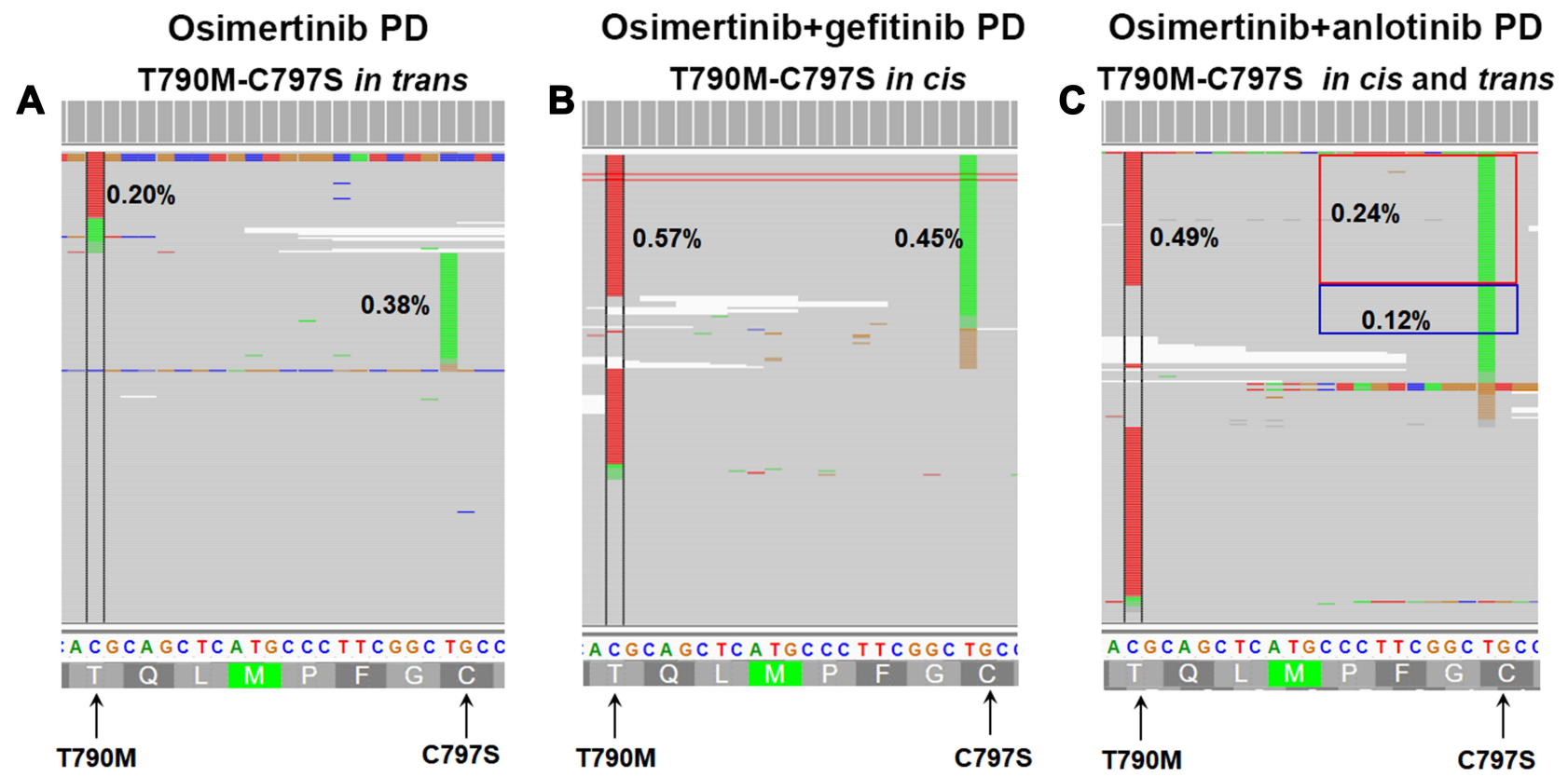

Figure 2 Allelic context and frequencies of EGFR C797S mutation and EGFR T790M mutation during treatments. (A) An in trans configuration at osimertinib PD (progressive disease); (B) an in-cis configuration at osimertinib+ gefitinib PD; (C) both in trans and cis configurations at osimertinib+anlotinib PD.

(Figure 1D). Only minimal side effects including paronychia and rash were observed.

After a PFS of more than 8 months, the disease progressed again. CT scan showed truncated bronchus in the right lower lobe, increased right pleural effusion, multiple nodules on the right lung and aggravated lung infection (Figure 1E). A repeated NGS test was performed with a plasma sample and showed EGFR 19del (AF; 0.62\%) and C797S (AF: 0.45\%) in cis-with T790M (AF: 0.57\%) (Figure 2B). The patient was subsequently treated with a combined regimen of osimertinib (80mg/QD), anlotinib (12mg/QD), pemetrexed (800mg), and cisplatin (30mg 13 day). After one cycle of treatment, she achieved a PR on the right lung lesion and reduction of pleural effusion. Right lung infection was also relieved. After two cycles of combined treatment, cisplatin was withdrawn due to severe gastrointestinal side effects. A repeated CT scan was performed in Dec 2019 and confirmed that the patient remained on PR (Figure 1F). She subsequently received osimertinib and anlotinib maintenance treatment until May 2020, when the disease progressed (Figure 1G), resulting in a PFS of 9 months. NGS was performed with the plasma sample at $\mathrm{PD}$ and revealed EGFR 19del (AF: $0.68 \%$ ) and C797S both in cis (AF: 0.24\%) and in trans (AF: 0.12\%) with T790M (AF: 0.49\%) (Figure 2C). The patient received a treatment of bevacizumab + albumin-bound paclitaxel beginning from June 2020. The symptoms of chest distress and shortness of breath were relieved, while the patient's mental status and appetite were improved. However, after two cycles, the treatment was terminated due to the poor physical condition of the patient. She passed away in August 2020 with an overall survival of 39 months.

\section{Discussion}

Our case demonstrated the shift of T790M-trans-C797S into in-cis configuration as a potential resistant mechanism to the combinational treatment of first- and secondgeneration TKIs, which has also been suggested by Wang et al. ${ }^{4}$ Currently, therapeutic options for NSCLC with T790M-cis-C797S remain scarce. Patients with this genomic profile are often treated with chemotherapy after osimertinib progression but with poor clinical outcomes. Recently, preliminary clinical evidence has shown the effectiveness of combinatorial treatment with dual-target anaplastic lymphoma kinase (ALK)-EGFR inhibitor brigatinib and cetuximab in this subset of NSCLC patients. ${ }^{6}$ In the study describing subsequent therapeutic management of the same patient reported by Wang et al, ${ }^{4}$ the regimen of chemotherapy plus bevacizumab resulted in a beneficial survival after the emergence of cis-C797S. ${ }^{7}$

In our study, the patient with EGFR 19del-T790M-cisC797S triple mutant achieved a PR lasting for 9 months to the combination of osimertinib, anlotinib, and chemotherapy, 
followed by osimertinib and anlotinib maintenance treatment. Since therapy was used in combination, we cannot pinpoint the exact response for either chemotherapy or osimertinib/anlotinib. However, our case suggests osimertinib + anlotinib maintenance treatment could provide benefit as indicated by the durable response of 5 months. Anlotinib is a novel multi-targeting tyrosine kinase (vascular endothelial growth factor receptor (VEGFR), fibroblast growth factor receptor (FGFR), platelet-derived growth factor receptor (PDGFR), and c-kit) inhibitor with a broad spectrum of inhibitory effects on tumor angiogenesis and growth. It has been approved in China for the treatment of patients with locally advanced or metastatic NSCLC after $\geq 2$ lines of systemic chemotherapy. ${ }^{8}$ Preclinical studies revealed an overactive VEGF/VEGFR pathway and tumor angiogenesis plays a crucial role in the resistance to EGFR-TKIs, and the dual targeting of both the VEGF and EGFR pathways may prevent resistance. 9,10 Currently, a Phase II study (NCT04438902) is ongoing which evaluates osimertinib combined with anlotinib in EGFR T790M mutated NSCLC patients with progression on osimertinib treatment. Our study provides the first clinical evidence for the potential efficacy of the combined therapy of osimertinib and anlotinib in overcoming the resistance mediated by cis-C797S.

In line with the case reported by Wang and $\mathrm{Wu}{ }^{7}$ trans-C797S re-emerged after the failure of treatment for cis-C797S in our case, resulting in the presence of T790M-C797S both in cis and trans. The heavily-treated patient subsequently received a combination of chemoand anti-angiogenic therapies. Although relief of symptoms was observed, she discontinued the treatment because of poor physical condition. Concomitant T790M$\mathrm{C} 797 \mathrm{~S}$ in trans and cis have been identified in $\sim 2 \%$ of C797S cases who progressed on osimertinib. ${ }^{5}$ Very limited studies have described the therapeutic information for this small subset of patients. And these reported therapies, including the combination of first- and thirdgeneration EGFR TKIs as well as anlotinib monotherapy, failed to achieve beneficial outcomes. ${ }^{7,11}$ Collectively, therapeutic options for NSCLC patients harboring concomitant T790M-C797S in trans and cis remain largely unknown thus require further investigation.

\section{Conclusion}

In conclusion, our study provides the first clinical evidence that the combination of osimertinib and anlotinib may be an effective therapy in overcoming resistance mediated by T790M-cis-C797S. Our case also highlights the importance of dynamically monitoring the mutation status after osimertinib failure, which may provide patients with increased opportunities for targeted therapy and improve postosimertinib progression survivals. ${ }^{3}$

\section{Data Sharing Statement}

All the data generated during this study are included in this published article. The datasets analyzed during the current study are available from the corresponding authors on reasonable request.

\section{Ethical Approval}

All procedures performed in studies involving human participants were in accordance with the ethical standards of the institutional and/or national research Committee(s) and with the 1964 Helsinki declaration and its later amendments or comparable ethical standard. Institutional approval was not required to publish the case details.

\section{Patient Informed Consent}

Written informed consent was obtained from the patient's family member for publication of this case report and any accompanying images in an anonymised manner.

\section{Disclosure}

The authors report no conflicts of interest in this work.

\section{References}

1. Camidge DR, Pao W, Sequist LV. Acquired resistance to TKIs in solid tumours: learning from lung cancer. Nat Rev Clin Oncol. 2014;11 (8):473-481. doi:10.1038/nrclinonc.2014.104

2. Thress KS, Paweletz CP, Felip E, et al. Acquired EGFR C797S mutation mediates resistance to AZD9291 in non-small cell lung cancer harboring EGFR T790M. Nat Med. 2015;21(6):560-562. doi:10.1038/nm.3854

3. Cheng JT, Yao YH, Gao YE, et al. Integrated histological and molecular analyses of rebiopsy samples at osimertinib progression improve post-progression survivals: a single-center retrospective study. Lung Cancer. 2020;150:97-106. doi:10.1016/j.lungcan.2020.10.010

4. Wang Z, Yang JJ, Huang J, et al. Lung adenocarcinoma harboring EGFR T790M and in trans C797S responds to combination therapy of first- and third-generation EGFR TKIs and shifts allelic configuration at resistance. $J$ Thorac Oncol. 2017;12(11):1723-1727. doi:10.1016/j. jtho.2017.06.017

5. Piotrowska Z, Nagy R, Fairclough S, et al. OA 09.01 characterizing the genomic landscape of EGFR C797S in lung cancer using ctDNA next-generation sequencing. $J$ Thorac Oncol. 2017;12(11):S1767. doi:10.1016/j.jtho.2017.09.375

6. Wang Y, Yang N, Zhang Y, et al. Effective treatment of lung adenocarcinoma harboring EGFR-activating mutation, T790M, and cis-C797S triple mutations by brigatinib and cetuximab combination therapy. $J$ Thorac Oncol. 2020;15(8):1369-1375. doi:10.1016/j. jtho.2020.04.014 
7. Wang $\mathrm{Z}, \mathrm{Wu} \mathrm{YL}$. Re-emerging $\mathrm{C} 797 \mathrm{~S}$ in trans and rechallenge of osimertinib with erlotinib. J Thorac Oncol. 2019;14(4):e81-e82. doi:10.1016/j.jtho.2018.12.015

8. Han B, Li K, Wang Q, et al. Effect of anlotinib as a third-line or further treatment on overall survival of patients with advanced non-small cell lung cancer: the ALTER 0303 Phase 3 Randomized Clinical Trial. JAMA Oncol. 2018;4(11):1569-1575. doi:10.1001/ jamaoncol.2018.3039

9. Masuda C, Yanagisawa M, Yorozu K, et al. Bevacizumab counteracts VEGF-dependent resistance to erlotinib in an EGFR-mutated NSCLC xenograft model. Int $J$ Oncol. 2017;51(2):425-434. doi:10.3892/ ijo.2017.4036
10. Yu Y, Wang Y, Wu L, et al. Efficacy of epidermal growth factor receptor tyrosine kinase inhibitors (EGFR-TKIs) combined with bevacizumab for advanced non-squamous non-small-cell lung cancer patients with gradual progression on EGFR-TKI treatment: a cohort study. Medicine (Baltimore). 2021;100(5):e23712. doi:10.1097/ MD.0000000000023712

11. Liu C, Li J, Liu H, et al. The concomitant EGFR T790M/C797S in trans and cis in three osimertinib-resistant lung adenocarcinoma patients. J Clin Oncol. 2019;37(15_suppl):e20105. doi:10.1200/ JCO.2019.37.15_suppl.e20105

\section{Publish your work in this journal}

OncoTargets and Therapy is an international, peer-reviewed, open access journal focusing on the pathological basis of all cancers, potential targets for therapy and treatment protocols employed to improve the management of cancer patients. The journal also focuses on the impact of management programs and new therapeutic

Submit your manuscript here: https://www.dovepress.com/oncotargets-and-therapy-journal agents and protocols on patient perspectives such as quality of life, adherence and satisfaction. The manuscript management system is completely online and includes a very quick and fair peer-review system, which is all easy to use. Visit http://www.dovepress.com/ testimonials.php to read real quotes from published authors. 